\title{
¿Qué papel juega la exposición solar en el desarrollo del cáncer de piel? \\ El efecto de la radiación ultravioleta en la acumulación de mutaciones somáticas en piel sana
}

\author{
Rocío Moreno Illueca \\ al298770@uji.es \\ Germán Herrero Rodríguez \\ al286668@uji.es \\ Bárbara Hernando Fuster \\ hernandb@uji.es \\ Conrado Martínez Cadenas \\ ccadenas@uji.es
}

\section{Resumen}

Introducción: El cáncer cutáneo surge a resultas de alteraciones genómicas en células de la piel, normalmente a consecuencia de la exposición a radiación solar. La detección de mutaciones somáticas en células normales puede revelar ideas clave sobre etapas iniciales del desarrollo de estos cánceres.

Objetivo: En este trabajo se pretende describir el número y el perfil de mutaciones somáticas de piel sana según el grado de exposición solar e inferir la influencia en este de factores como el genotipo pigmentario, la historia de exposición solar, la edad y las características fenotípicas del individuo.

Métodos: Se obtuvo ADN genómico de 93 epidermis sanas. Mediante secuenciación masiva se analizaron las mutaciones en 47 genes claves en el proceso tumoral. Se genotiparon variantes genéticas relacionadas con la sensibilidad solar. Se comparó la tasa mutacional y el perfil mutacional utilizando test no paramétricos (Mann-Whitney, Spearman o Kruskal-Wallis), con SPSSv25 y R.

Resultados: La tasa mutacional es mayor en epidermis expuesta al sol permanentemente y en individuos más ancianos. En cuanto al perfil mutacional, hay diferencias en el tipo de mutaciones y la firma mutacional según la exposición solar acumulada en el tejido. Individuos con un genotipo pigmentario asociado a tener una mayor sensibilidad solar no parecen tener mayor tasa mutacional, seguramente porque utilizan medidas preventivas. 
Conclusiones: La exposición solar acumulada en la piel es fundamental en el desarrollo del cáncer de piel ya que favorece la acumulación de mutaciones somáticas en estados preneoplásicos.

Palabras clave: radiación ultravioleta, mutaciones somáticas, cáncer de piel, melanoma, sensibilidad solar.

\section{Abstract}

Introduction: Melanoma arises as a result of alterations in the genomes of skin cells, usually as a consequence of exposure to sun radiation. The detection of somatic mutations in normal cells promises to reveal fundamental ideas in the early stages of skin cancer development.

Objective: To analyze the somatic mutational burden and profile of cancer-free skin according to the degree of sun exposure, and inferring the role of individual's history of sun exposure and their pigmentation-related phenotype and genotype in the accumulation of somatic mutations.

Methods: Genomic DNA was obtained from 93 cancer-free epidermises. Genetic variants associated with skin sensitivity to sunlight were genotyped. The mutations accumulated in 46 cancer-driver genes were analyzed by using next generation sequencing. The mutational burden and profile were compared by using nonparametric tests (Mann-Whitney, Spearman or Kruskal-Wallis), with the SPSSv25 and R.

Results: The somatic mutational burden was higher in epidermal samples from chronically-photoexposed body sites and in older individuals. Regarding the mutational profile, a different type of somatic mutations and mutational signature was observed among epidermal samples according to the degree of sun exposure accumulated in the cutaneous sample. Individuals carrying genetic variants associated with having a greater sunlight sensitivity do not seem to present a higher mutational rate, probably because they are more conscious about using preventive measures against sun damage.

Conclusion: The degree of sun exposure accumulated on the skin is fundamental in the development of skin cancer, as it significantly contributes to the accumulation of somatic mutations in preneoplastic states.

Keywords: ultraviolet radiation, somatic mutations, skin cancer, melanoma, solar sensitivity.

\section{Introducción}

El cáncer se define como una proliferación anormal de células, fundamentalmente debido a la adquisición de diferentes mutaciones somáticas que les confieren capacidad de supervivencia y replicación incontrolada (Basu 2018; Jorde, Carey y Bamshad 2011). La producción de una mutación somática suele ser al azar por la acción de factores dañinos externos y su reparación es básica para un funcionamiento normal de las células. A nivel de la patogénesis del cáncer, la no corrección y consiguiente acumulación de una serie de estas mutaciones en las células puede generar cambios en la función de la célula que afecten a genes claves en la supervivencia celular. Es importante tener en cuenta que el proceso carcinogénico conlleva un proceso evolutivo en el tiempo y se necesitan adquirir diversas mutaciones 
conductoras (del inglés driver mutations) en células fisiológicamente normales para que se desarrolle un tumor. De hecho, se han detectado mutaciones conductoras en células o tejidos fisiológicamente normales de sangre, esófago y piel (Risques y Kennedy 2018).

El cáncer de piel es una de las neoplasias más comunes en todo el mundo. De entre los cánceres cutáneos, el melanoma es el cáncer de piel con mayor relevancia clínica, tanto por su frecuencia como por su mortalidad (Bray et al. 2018). El melanoma es consecuencia de una proliferación incontrolada de los melanocitos, células localizadas en la capa más superficial de la piel que se encargan de la síntesis de melanina (pigmento que ejerce una acción protectora frente la radiación solar) (Schadendorf et al. 2018).

La predisposición a desarrollar un cáncer cutáneo está influenciada por numerosos factores, tanto de tipo ambiental (entre los que destacan la exposición a la luz solar, principal agente etiológico) como genético (variantes genéticas que determinan los rasgos de pigmentación cutánea del individuo). La exposición a la radiación ultravioleta (UV) es clave en la aparición del melanoma ya que produce alteraciones bioquímicas y estructurales en el ADN (acido desoxirribonucleico) de las células de la piel (Goldsmith et al. 2014b). Concretamente, la absorción de los fotones UV por el ADN es responsable de la generación de dos fotoproductos relevantes, los dímeros de pirimidina tipo ciclobutano (CPD y los fotoproductos de 6-4 pirimidina pirimidona (64PP). Ambas alteraciones se traducen en transiciones $C$ (Citosina) $\rightarrow T$ (Timina) y $\mathrm{CC} \rightarrow$ TT en las secuencias de dímeros de pirimidina (Basu 2018; Emri et al. 2018), constituyendo el patrón o firma mutacional característica de la mutagénesis inducida por la radiación UV (Alexandrov et al. 2013).

Otro factor de riesgo de cáncer de piel es el fototipo cutáneo del individuo, es decir, su tendencia a broncearse o a quemarse tras exponerse a la radiación solar. La sensibilidad cutánea a la radiación solar es un rasgo poligénico determinado por un gran número de polimorfismos localizados en genes relacionados con la síntesis de melanina, como por ejemplo el gen del receptor de la melanocortina 1 (MC1R), el de la proteína señalizadora de agouti $(A S I P)$, el de la basonucleína $2(B N C 2)$, el del segundo miembro de la familia 45 de trasportadores de soluto (SLC45A2) y el gen del factor 4 regulador del interferón (IRF4) (Goldsmith et al. 2014b).

Con todo ello, este trabajo pretende analizar el perfil de mutaciones somáticas acumuladas en piel fisiológicamente normal según el patrón de exposición a la radiación solar del tejido. Además, se analiza si existe un riesgo añadido según el genotipo pigmentario, la historia de exposición solar, la edad y las características fenotípicas del individuo.

\section{Métodos}

Se recogieron un total de 93 muestras cutáneas procedentes de pacientes distintos (46 hombres y 47 mujeres) y se obtuvo una muestra por paciente.

Además de obtener las muestras, los dermatólogos se encargaron de averiguar la información relevante para el estudio de cada participante. Para este fin, suministraron a los participantes un formulario estandarizado con el que recopilaron el sexo, la edad, los rasgos de pigmentación, el historial de quemaduras solares, la sensibilidad de la piel a la luz solar (fototipo cutáneo según Fitzpatrick) y los hábitos de exposición solar de los pacientes. Cada paciente completó el formulario bajo la supervisión del especialista. 
Las muestras de tejido se obtuvieron de las partes sobrantes de resecciones de piel realizadas para eliminar por escisión una lesión cutánea. La recogida de muestras la realizaron los especialistas de dermatología del Hospital General de Castellón y del Hospital La Plana de Vila-real. Del total de muestras recogidas, $2(2,3 \%)$ pertenecían a zonas no fotoexpuestas (axila y nalgas), 55 (62,5\%) eran de zonas intermitentemente expuestas al sol (tronco, espalda, hombro y parte superior de las extremidades) y $31(35,2 \%)$ provenían de zonas crónicamente expuestas a la radiación solar (cara, cuello, antebrazos y dorso de las manos).

Inmediatamente tras la resección tisular, todas las muestras cutáneas se sumergieron en la solución RNAlater para estabilizar el material biológico (Thermo Fisher Scientific, Walham, MA, EE. UU.). Una vez en el laboratorio, las piezas de tejido fueron sumergidas en tiocinato de amonio al 3,8 \% (Sigma-Aldrich, St. Louis, MO, EE. UU.) en solución salina tamponada con fosfato $(\mathrm{pH} 7,4)$ y se incubaron durante 3 horas a temperatura ambiente para separar la epidermis de la dermis. Las extracciones de ADN de las epidermis obtenidas se realizaron utilizando el QIAamp DNA Mini Kit (Qiagen, Hilden, Alemania), siguiendo las recomendaciones del fabricante.

Para identificar las mutaciones somáticas en las biopsias de piel, se realizó una secuenciación dirigida de los exones de un panel de 47 genes seleccionados por: 1) ser genes involucrados en diferentes tumoraciones y tipos de cáncer de piel (nevus, melanoma, carcinoma basocelular, carcinoma espinocelular) (Jayaraman, Rayhan, Hazany y Kolodney 2014), 2) ser genes que están involucrados en la progresión tumoral de una amplia gama de tipos neoplásicos (Davies et al. 2002) o 3) ser genes mutados con frecuencia en muestras de piel normal (Martincorena et al. 2015). La secuenciación de los genes diana se realizó utilizando tecnología de secuenciación de nueva generación (NGS), en el Centro de Regulación Genómica de Barcelona (CRGCNAG). Las secuencias obtenidas para cada muestra se compararon con la secuencia consenso de cada uno de los genes diana analizados disponibles en la base de datos GenBank (http://www.ncbi.nlm.nih.gov/genbank/). En esta comparación se pretendía eliminar aquellas mutaciones presentes en línea germinal, suprimiendo aquellas variantes que tuvieran una frecuencia en la población española mayor al $1 \%$, según la base de datos Ensembl (www.ensembl.org). Los análisis bioinformáticos de filtrado de variantes se llevaron a cabo en el CRG-CNAG por el Departamento de Análisis de Datos.

Debido a la importancia del genotipo pigmentario en la predisposición a cáncer cutáneo, se analizó el genotipo de genes clave en la sensibilidad cutánea a la radiación solar en cada uno de los participantes. El análisis genético del gen $M C 1 R$ se realizó mediante la secuenciación completa de la región codificante del gen, utilizando la tecnología de secuenciación Sanger. Además, se genotiparon variantes genéticas localizadas en los genes ASIP, BNC2, SLC45A2 e IRF4. Todas las reacciones de genotipado se realizaron utilizando KASP SNP Genotyping Chemistry (LGC, Hoddesdon, Reino Unido), siguiendo las recomendaciones del fabricante.

El análisis de los datos se realizó utilizando los programas SPSSv.25 (IBM Corp. Released 2017. IBM SPSS Statistics for Windows, Version 25.0. Armonk, NY: IBM Corp.) y RStudio (RStudio Team; 2019. RStudio: Integrated Development for R. RStudio, Inc., Boston, MA). La prueba de Saphiro-Wilk se empleó para comprobar la distribución normal de las variables cuantitativas. Al no seguir una distribución normal, los análisis estadísticos se llevaron a cabo utilizando diferentes pruebas no paramétricas. Se utilizó el test de $U$ de Mann-Whitney para comparar una variable cuantitativa entre dos muestras independientes y el test de Kruskal-Wallis para realizar las comparaciones entre tres muestras independientes. La correlación de Spearman 
se utilizó para determinar la relación lineal entre dos variables cuantitativas. La prueba Chi cuadrado de Pearson se empleó para comparar variables categóricas entre grupos. El nivel de confianza estadística para establecer diferencias significativas fue del $95 \%$.

\section{Resultados}

En primer lugar, se analizó si existían diferencias en el número de mutaciones somáticas que presentaba el tejido resecado en función de su grado de exposición solar acumulada (figura 1). Los datos permiten afirmar que existe una diferencia significativa en la cantidad de mutaciones en función de la exposición al sol de los tejidos epidérmicos ( $p$-valor $<0,001)$ y es mayor en aquellas muestras cutáneas resecadas de una zona corporal expuesta al sol de forma permanente. La carga mutacional acumulada es estadísticamente significativa entre las muestras con exposición solar intermitente y crónica ( $p$-valor $<0,001)$, así como entre las muestras no fotoexpuestas y aquellas crónicamente expuestas al sol ( $p$-valor=0,006). Por el contrario, no se encuentran diferencias significativas en el número de mutaciones acumuladas en muestras expuestas de forma intermitente y aquellas no fotoexpuestas ( $p$-valor=0,215), probablemente por el escaso número de muestras que se recogieron de zonas ocultas a la exposición solar.

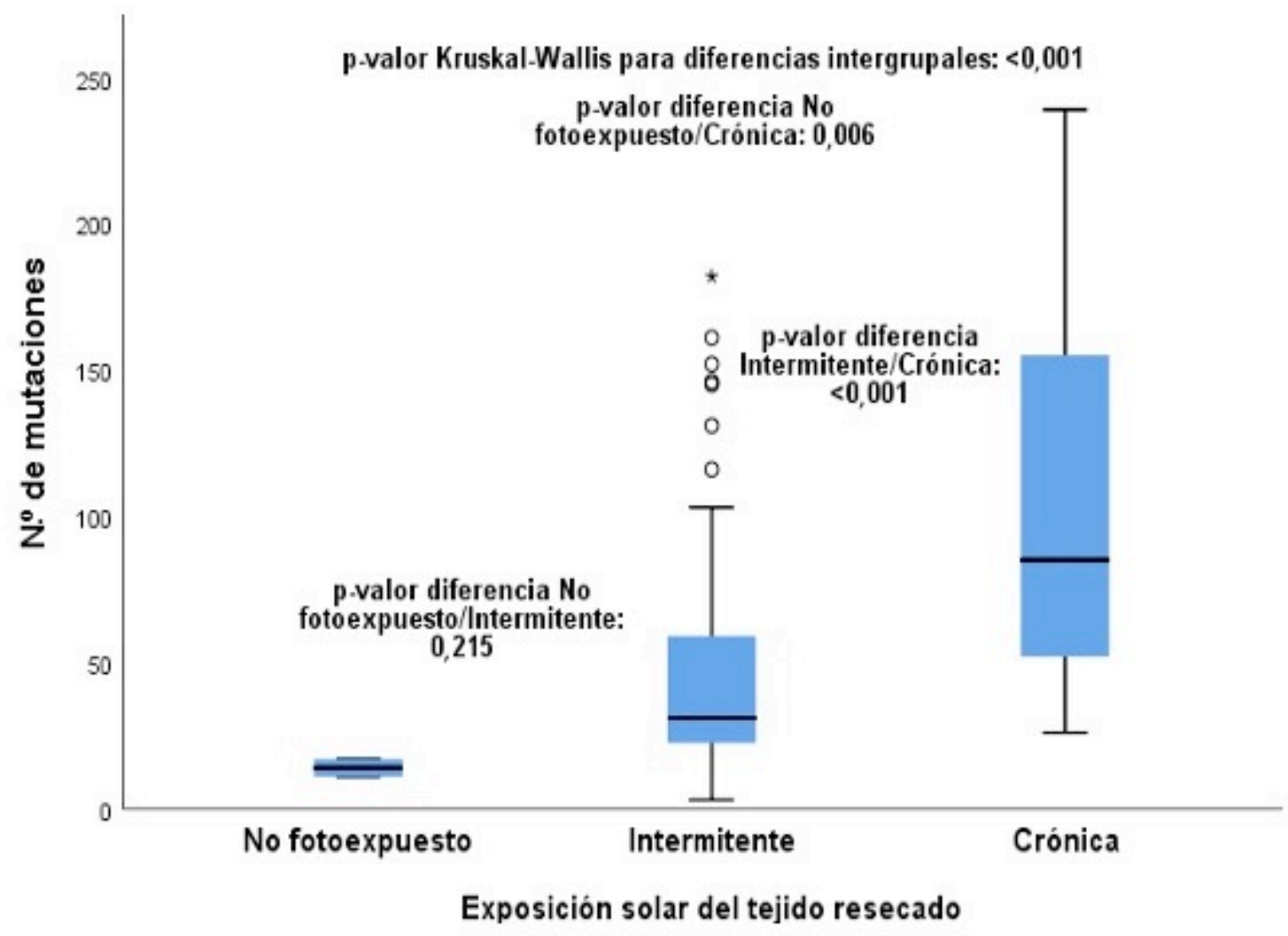

Figura 1. Representación gráfica de la cantidad de mutaciones somáticas acumuladas según la exposición solar del tejido resecado.

En segundo lugar, se analizaron las diferencias en la proporción de cambios nucleotídicos según el patrón de exposición solar del tejido resecado, con el objetivo de analizar si había diferencias en el perfil mutacional dependiendo de la exposición 
solar acumulada en la muestra (figura 2A). El análisis de estos datos permite establecer la existencia de diferencias estadísticamente significativas según el patrón de exposición solar del tejido para los cambios nucleotídicos $C>T$ ( $p$-valor=0,012), siendo mayor la proporción de transiciones $\mathrm{C}>\mathrm{T}$ en aquellas zonas con mayor exposición solar. Además, se encuentran diferencias en la frecuencia de cambios nucleotídicos $T>C$ ( $p$-valor $=0,017$ ), siendo la proporción de este cambio nucleotídico en aquellas zonas de tejido no expuesto a la radiación solar.

Por otra parte, se analizó la proporción de mutaciones somáticas que afectaban a zonas codificantes y no codificantes según el grado de exposición solar del tejido (figura 2B). Los resultados muestran diferencias significativas en la proporción de mutaciones acumuladas en regiones codificantes $(p$-valor $=0,014)$ entre grupos de exposición solar. Se observa que las mutaciones somáticas afectan en mayor medida a zonas codificantes en aquellas muestras cutáneas expuestas de forma crónica al sol. Así pues, las muestras cutáneas expuestas al sol tienen un mayor número de mutaciones somáticas y, además, estas suelen acumularse en zonas del genoma que codifican para una proteína. En cambio, los tejidos que no se exponen a la radiación solar tienden a acumular las pocas mutaciones somáticas que tienen presentes en zonas no codificantes del genoma.

(A)

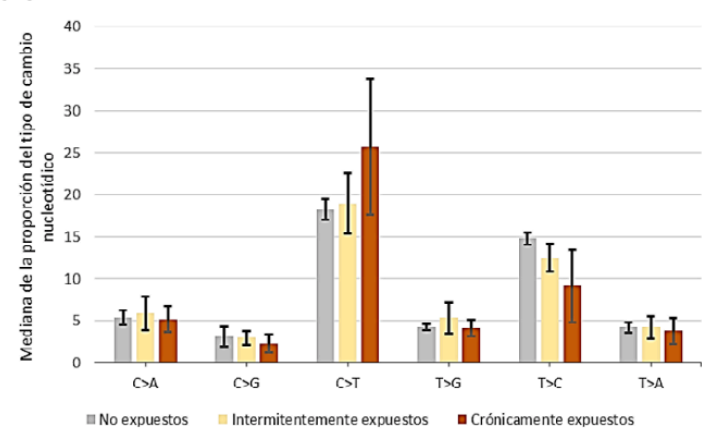

(B)

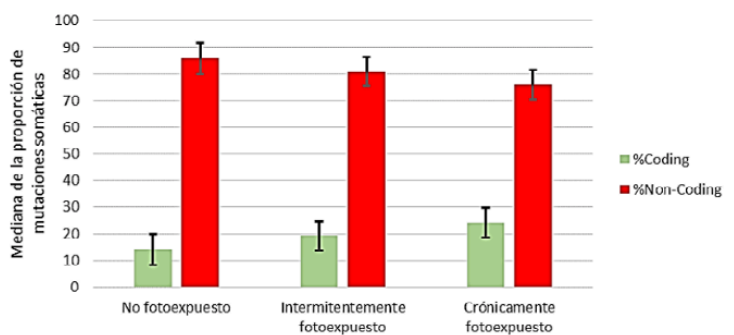

Figura 2. Representación de la distribución de mutaciones somáticas según la exposición del tejido por (A) tipo de cambio nucleotídico y (B) la región del genoma dónde se acumulan (zonas codificantes o no codificantes). Cada barra representa la mediana y el rango intercuartílico.

Seguidamente, se estudió si existía un mayor número de mutaciones somáticas acumuladas en los tejidos cutáneos a mayor edad del individuo (figura 3). Tal y como se esperaba, se observa una correlación positiva entre la edad y la carga mutacional del tejido resecado $(\rho=0,573, p$-valor $=8,3937 \mathrm{E}-9)$ : a mayor edad $y$, por lo tanto, a mayor tiempo expuesto a la radiación UV, más mutaciones somáticas se acumulan en la piel. 


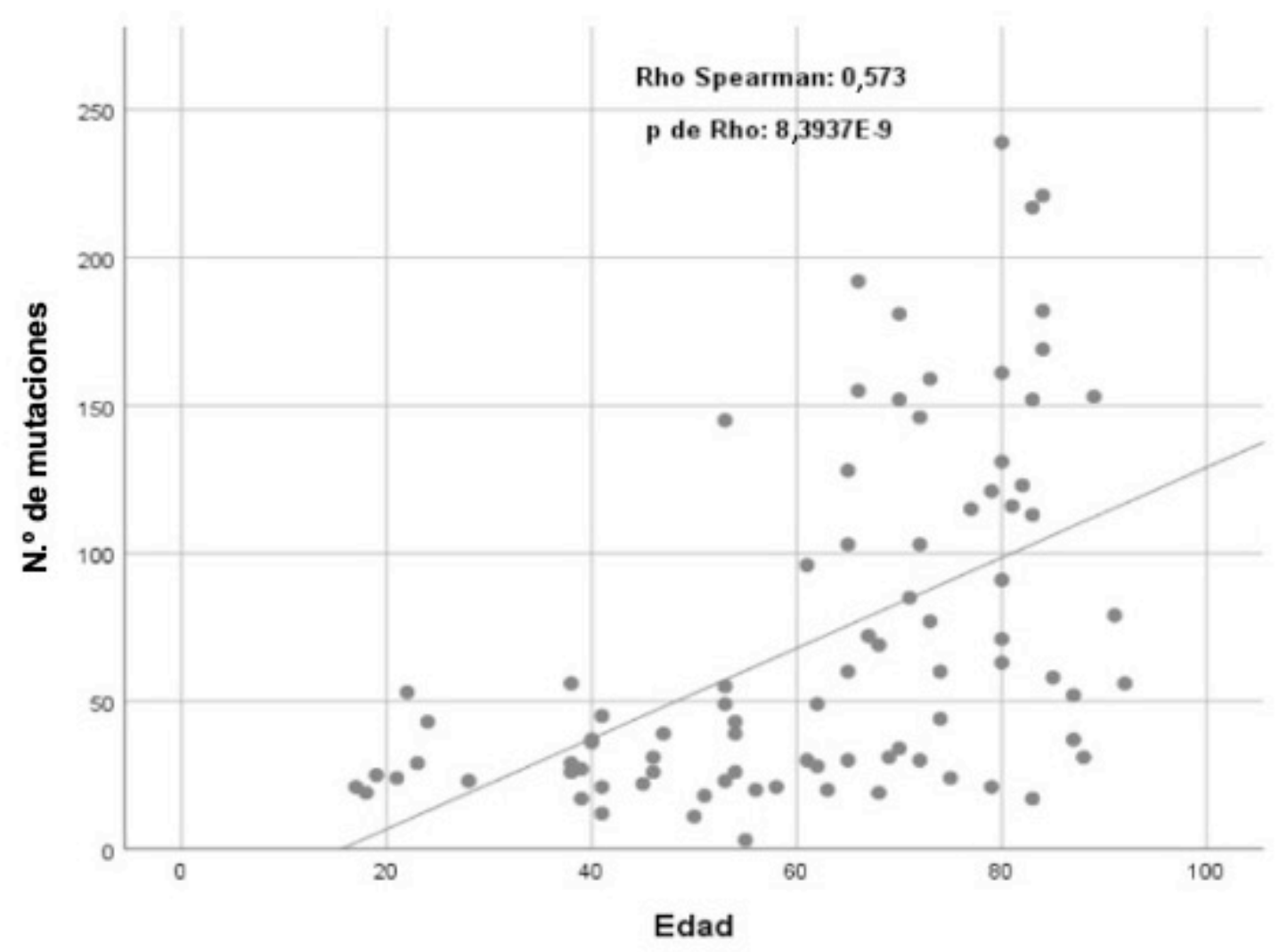

Figura 3. Representación gráfica de la relación existente entre la edad del sujeto y la cantidad de mutaciones somáticas que presenta su epidermis.

A continuación, se analizó si tener rasgos fenotípicos de la pigmentación relacionados con la sensibilidad solar es un riesgo añadido a acumular mutaciones somáticas en genes clave en el proceso tumoral y, por lo tanto, a desarrollar un cáncer de piel. Según los resultados obtenidos, no se observan diferencias significativas en el número de mutaciones acumuladas según el fototipo cutáneo de Fitzpatrick del individuo, tanto en muestras no crónicamente expuestas al sol ( $p$-valor $=0,320$ ) como en aquellas fotoexpuestas de forma crónica ( $p$-valor $=0,711$ ). Tampoco parece existir una asociación entre el color de la piel basal y la cantidad de mutaciones somáticas acumuladas en el tejido cutáneo, tanto en muestras sin exposición crónica ( $p$ valor $=0,531$ ) como en aquellas expuestas de forma intermitente ( $p$-valor=0,478). La presencia de efélides, rasgo de sensibilidad solar, tampoco parece influir en la carga mutacional de las muestras epidérmicas, tanto en muestras sin exposición crónica al sol ( $p$-valor $=0,779$ ) como en aquellas muestras de zonas corporales expuestas de forma permanente al sol ( $p$-valor $=0,678)$.

Por último, se decidió analizar el papel del genotipo pigmentario del individuo. Tener un perfil genotípico de riesgo no parece influir significativamente en la acumulación de mutaciones somáticas en piel. Al estudiar de forma independiente el genotipo del gen $M C 1 R$ (gen clave en la respuesta a la radiación ultravioleta), tampoco se encuentran diferencias entre los individuos portadores de mutaciones de pérdida de función (variantes R) y aquellos portadores de mutaciones que disminuían la función del receptor (variantes $r$ ) respecto a los individuos wild-type o normales. La ausencia de diferencias significativas se observa tanto en muestras expuestas al sol de forma 
intermitente ( $p$-valor $=0,896)$ como en aquellas muestras cutáneas de zonas expuestas crónicamente al sol ( $p$-valor=0,829).

\section{Discusión y conclusiones}

Este estudio se centra en ampliar el conocimiento actual respecto a los mecanismos genéticos y moleculares que llevan a las células cutáneas normales a evolucionar a células tumorales y ver si los factores de riesgo asociados al cáncer de piel influyen en el tipo y el número de mutaciones somáticas acumuladas en la piel.

El primer objetivo planteado en este trabajo fue comparar la cantidad de mutaciones somáticas acumuladas en las muestras de piel según el patrón de exposición solar del tejido resecado. Los resultados obtenidos muestran la existencia de diferencias significativas en la carga mutacional dependiendo del patrón de exposición al sol de cada muestra analizada, siendo mayor en muestras cutáneas resecadas de zonas corporales que se encuentran crónicamente expuestas al sol. Estudios previos ya identificaban una mayor carga mutacional en genes relacionados con el proceso tumoral en aquellas células de melanomas cutáneos expuestos al sol, en comparación con aquellos melanomas que surgen en zonas ocultas al sol, como el melanoma mucoso y acral (Hayward et al. 2017).

Estos resultados, junto con el hecho de que las muestras con un mayor grado de exposición solar presentaban una prevalencia de cambios $\mathrm{C}>\mathrm{T}$ en su material genético, confirman el papel fundamental que juega la radiación solar en la alteración del material genético de las células epidérmicas. Tal y como se explica en la introducción, la transición C>T es típica de la acción dañina que provoca en el ADN la radiación UV (Goldsmith et al. 2014a). Al analizar el contexto de estos cambios nucleotídicos se puede discernir que las firmas mutacionales más comunes en las muestras cutáneas analizadas son las firmas mutacionales 7,11 y 23 (descritas en la base de datos de referencia COSMIC, https://cancer.sanger.ac.uk/cosmic/signatures). Estas firmas mutacionales se han asociado a la radiación UV y son comúnmente observadas en cánceres de piel y labio. La presencia de esta firma mutacional en muestras cutáneas normales expuestas al sol de forma crónica o intermitente confirmaría nuestra hipótesis inicial en la que sospechábamos que la radiación UV es el factor etiológico principal en la aparición de mutaciones somáticas en tejidos cutáneos.

Además, es interesante que las muestras obtenidas de zonas corporales no expuestas al sol (nalgas y axilas) parecen tener otro perfil mutacional, ya que presentan un mayor número de cambios nucleotídicos $\mathrm{T}>\mathrm{C}$ en comparación con las muestras expuestas crónicamente o intermitentemente a la radiación solar. Analizando la firma mutacional relacionada con este cambio nucleotídico, se ha observado que son firmas presentes en numerosos tipos de cáncer en órganos internos donde la radiación solar tiene poco papel como factor etiológico, según la base de datos COSMIC.

Después de analizar el perfil de las mutaciones somáticas acumuladas, es importante conocer en qué parte del genoma se acumulan estas mutaciones para ver si tienen transcendencia en el funcionamiento correcto de la célula epidérmica. Para ello, se calculó el porcentaje de mutaciones somáticas acumuladas en zonas codificantes y no codificantes respecto al total en cada muestra. Así, se ha podido observar que existe un incremento de la proporción de mutaciones somáticas en zonas codificantes del genoma cuanta mayor exposición solar presenta la muestra, en detrimento de las mutaciones acumuladas en zonas no codificantes. Las mutaciones somáticas en zonas codificantes del genoma producen una alteración a nivel estructural y funcional 
de las proteínas que codifican. Como los genes analizados son clave en la oncogénesis, la acumulación de estas mutaciones puede suponer una ventaja en la supervivencia celular y, por lo tanto, ser seleccionadas y acumularse así en ese tejido.

Por otro lado, son varios los estudios que han señalado que la carga mutacional incrementa con la edad en tejidos sanos sin carácter preneoplásico (Risques y Kennedy 2018). Además, el hecho de que el proceso carcinogénico sea un proceso evolutivo en el tiempo que conlleva la adquisición de varias mutaciones conductoras explicaría por qué la mayoría de los cánceres esporádicos ocurren en individuos de edad avanzada. Como era de esperar, nuestros resultados indican una mayor cantidad de mutaciones somáticas en muestras procedentes de individuos de mayor edad y, por tanto, han estado más tiempo expuestas a la acción lesiva de la radiación solar.

Por último, se analizó la influencia de ciertos rasgos de pigmentación (fototipo cutáneo, color de piel y presencia de efélides) en la acumulación de mutaciones somáticas, así como el perfil genético de sensibilidad solar del individuo. Se esperaba encontrar una mayor carga mutacional en las muestras epidérmicas procedentes de individuos con una mayor sensibilidad cutánea a la radiación UV, aunque ninguna de las características evaluadas parece influir en la tasa mutacional según los resultados obtenidos en nuestra población de estudio. Una posible explicación para esta falta de asociación, ya expuesta en estudios anteriores (Diao y Lee 2013), reside en que aquellos individuos que tienen una mayor sensibilidad solar (pelirrojos, pecosos, piel blanca) sean más conscientes del daño causado por la radiación ultravioleta y, por lo tanto, adopten medidas preventivas en mayor medida que estudios anteriores han establecido. En este estudio no se tuvieron en consideración conductas socioculturales relacionadas tangencialmente con los hábitos de exposición solar (empleo, uso de fotoprotector, vestimenta, etc.), aspecto que se debería incluir en el formulario para futuros estudios.

Tampoco parece influir en la acumulación de mutaciones somáticas tener un perfil genético asociado a una mayor sensibilidad cutánea al sol. Sin embargo, un estudio previo indica que melanomas de individuos portadores de variantes de pérdida de función en el gen $M C 1 R$ presentaban una mayor carga mutacional (Robles-Espinoza et al. 2016). Sería de esperar que individuos que no fueran capaces de sintetizar eumelanina, debido a la no funcionalidad del receptor MC1R, acumularan más alteraciones en su genoma ya que, como se ha comentado en la introducción, la eumelanina es el pigmento encargado de absorber los rayos UV, evitando así que incidan sobre el ADN genómico de las células epidérmicas.

El reducido número de individuos con variantes de pérdida de función en $M C 1 R$ en España, y por tanto en nuestra población, podría ser la causa de que no obtuvieran resultados significativos.

En conclusión, este trabajo confirma que la exposición solar acumulada en la piel es clave en el desarrollo del cáncer cutáneo ya que favorece la acumulación de mutaciones somáticas en estados preneoplásicos. Además, se observa que el perfil de mutaciones somáticas que favorecerían la aparición de un cáncer cutáneo parece ser distinto según la zona corporal. Estos resultados pueden ser relevantes en un futuro de cara a la prevención de la progresión de estas lesiones en el ADN a lesiones precancerosas o cancerosas. 


\section{Referencias bibliográficas}

Alexandrov, Ludmil B., Serena Nik-Zainal, David D. Wedge, Samuel A. J. R. Aparicio, Sam Behjati, Andrew V. Biankin y Michael Stratton. 2013. "Signatures of mutational processes in human cancer». Nature, 500(7463): 415-421. https://doi.org/10.1038/nature12477

Basu, Asis K. 2018. «DNA Damage, Mutagenesis and Cancer». International Journal of Molecular Sciences, 19(4): 970. https://doi.org/10.3390/ijms19040970

Bray, Freddie, Jacques Ferlay, Isabelle Soerjomataram, Rebecca L. Siegel, Lindsey A. Torre y Ahmedin Jemal. 2018. "Global cancer statistics 2018: GLOBOCAN estimates of incidence and mortality worldwide for 36 cancers in 185 countries». CA: A Cancer Journal for Clinicians, 68(6): 394-424. https://doi.org/10.3322/caac.21492

Davies, Helen, Graham R. Bignell, Charles Cox, Philip Stephens, Sarah Edkins, Sheila Clegg y Andrew Futreal. 2002. "Mutations of the BRAF gene in human cancer». Nature, 417(6892): 949-954. https://doi.org/10.1038/nature00766

Diao, Diana Y. y Tim K Lee. 2013. "Sun-protective behaviors in populations at high risk for skin cancer». Psychology Research and Behavior Management, 7: 9-18. https://doi.org/10.2147/PRBM.S40457

Emri, Gabriella, György Paragh, Ágnes Tósaki, Eszter Janka, Sándor Kollár, Csaba Hegedüs y Éva Remenyik. 2018. "Ultraviolet radiation-mediated development of cutaneous melanoma: An update». Journal of Photochemistry and Photobiology B: Biology, 185: 169-175. https://doi.org/10.1016/j.jphotobiol.2018.06.005

Goldsmith, Lowell A., Stephen I. Katz, Bárbara A. Gilchrest, Amy S. Paller, David J. Leffell y Klaus Wolff. 2014a. "Carcinogénesis». En Fitzpatrick. Dermatología en Medicina General (vol. 1). Editorial Médica Panamericana.

Goldsmith, Lowell A., Stephen I. Katz, Bárbara A. Gilchrest, Amy S. Paller, David J. Leffell y Klaus Wolff. 2014b. "Tumores melanocíticos». En Fitzpatrick. Dermatología en Medicina General. Editorial Médica Panamericana.

Hayward, Nicholas K., James S. Wilmott, Nicola Waddell, Peter A. Johansson, Mathew A. Field, Katia Nones y Graham J. 2017. «Whole-genome landscapes of major melanoma subtypes». Nature, 545(7653): 175-180.

https://doi.org/10.1038/nature22071

Jayaraman, Shyam S., David J. Rayhan, Salar Hazany y Michael S. Kolodney. 2014. "Mutational landscape of basal cell carcinomas by whole-exome sequencing». The Journal of Investigative Dermatology, 134(1): 213-220. https://doi.org/10.1038/jid.2013.276

Jorde, Lynn B., John C. Carey y Michael J. Bamshad. 2011. Genética Médica. Ámsterdam: Elsevier.

Martincorena, Iñigo, Amit Roshan, Moritz Gerstung, Peter Ellis, Peter Van Loo, Stuart McLaren y Peter J. Campbell. 2015. «Tumor evolution: High burden and pervasive positive selection of somatic mutations in normal human skin». Science (New York, N.Y.), 348(6237): 880-886. https://doi.org/10.1126/science.aaa6806

Risques, Rosa Ana y Scott R. Kennedy. 2018. "Aging and the rise of somatic cancerassociated mutations in normal tissues». PLoS Genetics, 14(1): e1007108. https://doi.org/10.1371/journal.pgen.1007108

Robles-Espinoza, Carla Daniela, Nicola D. Roberts, Schuyang Chen, Finbarr P. Leacy, Ludmil B. Alexandrov, Natapol Pornputtapong y David J Adams. 2016. "Germline MC1R status influences somatic mutation burden in melanoma». Nature Communications, 7: 12064. https://doi.org/10.1038/ncomms12064

Schadendorf, Dirk, Alexander C. J. van Akkooi, Carola Berking, Klaus G. Griewank, Ralf Gutzmer, Axel Hauschild y Selma Ugurel. 2018. "Melanoma». Lancet (London, England), 392(10151): 971-984. https://doi.org/10.1016/S0140-6736(18)31559-9 